\title{
An ethnobotanical survey of medicinal and other useful plants of Muruts in Sabah, Malaysia
}

\author{
Julius Kulip
}

\begin{abstract}
Kulip, J. (Ethnobotany Section, Forest Research Centre, Forestry Department Sabah, P.O. Box 1407, 90715 Sandakan, Sabah, Malaysia) 2003. An ethnobotanical survey of medicinal and other useful plants of Muruts in Sabah, Malaysia. Telopea 10(1): 81-98. This paper presents the results of ethnobotanical studies on medicinal and other useful plants used traditionally by the Muruts in Sabah. They have rich ethnobotanical resources. A total of 91 species of plants were noted and collected during the surveys. Among them are 68 species of medicinal plants and 64 species of other useful plants. Reports from plant informants were obtained during field studies in February 1994, in March 1998, in May 1998, in May 2000 and in February 2001. For each species are given the botanical name, Murut name(s), collection number, medicinal uses, as well as plant part(s) used, and other uses. The popularity of plants with traditional uses among the Muruts is fading due to migration, restriction from religion, lost interest of younger generations and heavy dependence on modern medicine. Opening of forests for agricultural development and timber harvesting makes the resources scarce which is also contributing to the loss of knowledge. Awareness activities in Malaysia's primary and secondary schools on the usefulness of plants and their environment, and government policies on medicinal plants, have contributed significantly towards the preservation of indigenous traditional knowledge of plants.
\end{abstract}

\section{Introduction}

Sabah is one of the 13 states within the Federation of Malaysia and is located in the northernmost part of Borneo. It is the second largest state in Malaysia with a landmass of approximately 7.4 million hectares. The total forested area is 4.7 million hectares. The climate is marine equatorial with an average temperature of $74-88^{\circ} \mathrm{F}\left(23-32^{\circ} \mathrm{C}\right)$. The rainy season, locally called 'Musim hujan', is around November to February. This is also known as the North-east Monsoon. The South-west Monsoon, which is less wet, is from May to October, making the annual rainfall 60-120 inches (1525-3050 mm, or sometimes up to $4,500 \mathrm{~mm}$ ). Sabah, being part of Borneo, is rich in plant biodiversity. There is also an abundance of medicinal plants and other plants for everyday use.

Population in Sabah as at 2000 is about 3.0 million (Dept. Statistics 2000). There are 36 indigenous groups livng in Sabah; the Murut population is estimated at 91,700 and is the third largest. They are distributed in Keningau, Tenom, Nabawan/Pensiangan and Kalabakan districts. These districts are located in the interior and south-eastern parts of Sabah and the territory straddling the borders of Kalimantan (Indonesia) and Sarawak (Fig. 1). The definition of Muruts people adopted here is based on Tombung (1991) and King (1992). 
Survey work on Muruts' ethnobotanical resources on medicinal plants and other useful plants in Sabah began in early 1991 by the Sabah Museum and Universiti Kebangsaan Malaysia, Sabah Campus, while at the Forestry Department it began formally in 1999 even though collection of materials started in early 1994. A medicinal plant survey by Guntavid (1992) found that 38 species of plants were used by the Tangala Muruts in Kampung (Kg.) or village Inarad, Ulu Kinabatangan (upper Kinabatangan river). Fasihuddin and Hasmah (1992) found 57 species of plants were used as medicines by Muruts around Nabawan, Sepulut and Pensiangan area.

Medicines, or Tatapis (Paste)/Babas (antidote) in Muruts' language, from plant resources have been used for a long time by the Murut society to cure their everyday ailments. There is an equally long history of the use of wild plants for food, construction, house utensils, and social and religious functions. In the olden days herbal remedies provided the only relief when modern medicines were not available. Knowledgeable elders or medicine men usually administered the medicine. Depending on the ailments, the healing would often involve some kind of ritual. To the Muruts, the river and the forest represent the world. From them they derive their food, their medicine, their clothing and their house materials.

This paper presents the results of ongoing ethnobotanical research on the Murut people in Sabah. Its purpose is to preserve the precious cultural heritage of the Murut ancestors' use of plants. It is also hoped that further studies on the phytochemical contents of the plants will reveal some useful drugs for the benefit of mankind.

\section{Location and Area of Study}

The surveys focus on the various Muruts villages throughout Sabah (Fig. 2). Murut ethnic groups involved in these studies were Keningau Murut, Timugon Murut, Paluan Murut, Kalabakan Murut and Tagal Murut. Villages involved include Kg. (village) Melalap in Tenom district (Timugon Murut); Kg. Melinja and Bahagia B in Nabawan/Pensiangan district (Paluan Murut); Kg. Lingawon in Keningau district (Keningau Murut); Kg. Ulu Kalabakan in Kalabakan district (Kalabakan Murut); and Kg. Murni Empat and Enam in Nabawan/ Pensiangan district (Tagal Murut).

$\mathrm{Kg}$. Melalap is located about $15 \mathrm{~km}$ from Tenom town center and consists of about 100 households; Kg. Melinja and Bahagia B are located about five $\mathrm{km}$ from Nabawan town center and consists of about 100 households; $\mathrm{Kg}$. Lingawon is located about $10 \mathrm{~km}$ from Keningau town center and consists of about 50 households; Kg. Ulu Kalabakan is located about three $\mathrm{km}$ from Kalabakan town center and consists of about 50 households; and Kg. Murni Empat and Kg. Enam are located one km from Nabawan town center.

Ethnic groups such as the Murut turn to the forest for their daily needs and also for income. Produce such as wild vegetables, fruits and medicinal plants are gathered and sold in the weekly markets or 'Tamu' for an additional income.

The original vegetation cover of the research area can be classified as lowland mixed dipterocarp forests except for the Pensiangan area. A wet season usually begins in September and can last to January. At higher elevations such as in Pensiangan area, the forest is dominated by species of Fagaceae, Araucariaceae (Agathis spp.) and Myrtaceae, while at lower elevations such as in Tenom, Keningau and Kalabakan, species of Dipterocarpaceae, Leguminosae and Euphorbiaceae species predominate. 


\section{Methods}

Written permission from the office of the District Community Development and verbal permission from the village Chief concerned were usually applied for before entering any villages. Data were gathered by interviewing the plant informant(s), usually the knowledgeable villagers of Muruts elders (men and women) together with some young people who still used plants in their daily life. The interviews were conducted in Murut, which was spoken by all of the members of the Murut communities. The interview process was based on methods described by Martin (1995). Usually after a short interview, the plant informants would take us to their ladang (upland agricultural field), backyard gardens and into the forest. Fieldwork was carried out by a team of Ethnobotany Section staff from the Forest Research Centre of the Forestry Department Sabah, Sandakan.

Voucher specimens are deposited at the Ethnobotany Office at the Forest Research Centre Sabah, Forestry Department Sabah, Sandakan. Specimens that were easily identified in the field were not collected, only noted. Unidentifiable specimens were numbered and brought to the herbarium to be examined further.

Villages involved in these studies were namely $\mathrm{Kg}$. Melalap on 1-15 ${ }^{\text {th }}$ February 1994 where we interviewed Madam Kasium Galawang (62 years); Kg. Melinja and Kg. Bahagia B on 9-23 ${ }^{\text {rd }}$ March 1998 where we interviewed Mr. Kumalu A. (60 years), Mr. Lasuan Liwau (45 years) and Mr. Muskin Bugan (60 years); Kg. Lingawon on 6-18 ${ }^{\text {th }}$ May 1998 where we interviewed Madam Murika Gambun (55 years); Kg. Ulu Kalabakan on 8-17th May 2000 where we interviewed Mr. Mantawasa Baukom (60 years); and Kg. Murni Empat and Enam on 14-26 $6^{\text {th }}$ February 2001 where we interviewed Mr. Lintong Eman (45 years). A Murut language translator was used during the surveys. Most interviews lasted four to five hours per day. According to their Native Customary Law, a person must give to the plant informant a pingkaras or a kind of compensation. The compensation is normally one black hen and a sum of (RM 20.00) per species of plants. For this survey a sum of RM 20.00 (US\$ 5) was paid per day to the informants as a token of appreciation for their kind cooperation during the survey.

\section{Results}

Table 1 and Table 2 show the list of medicinal and other useful plants collected during the surveys. In total 91 species of plants were documented. Of these, 68 are indicated by Muruts informants as medicines, 64 as other useful plants (some of which were also used as medicines). Compared to previous collections by authors like Guntavid (1992) and Fassihuddin and Hasmah (1992), there are an additional 50 new records of medicinal plants collected during the present survey. The total number of Murut medicinal plants documented so far for Sabah, amounts to 143 species (Appendix 1) belonging to 44 families. Most prominent among plant families used by the Murut are Euphorbiaceae (10 species documented), followed by Leguminosae (6), Rubiaceae (5) (Appendix 2). There are 94 species found to be indigenous while 34 are introduced, naturalised, or only known from cultivation.

Plants which have a very high value, whether for medicine or other uses, are collected from the forest and often recultivated near the houses or in gardens so that there is a ready supply of the raw materials for future use. That is why many of these plants are quite commonly found in the village. A number of other plants however, can be found in nearby ladang (upland agricultural fields), disturbed forest or in relatively undisturbed mixed dipterocarp forest and roadsides. 
TABLE 1. PLANTS TRADITIONALLY USED AS MEDICINES BY THE MURUTS COMMUNITIY IN SABAH, MALAYSIA

Botanical name and voucher specimen no. (if any)

\section{ACANTHACEAE}

*Justicia gendarussa L.

\section{AGAVACEAE}

Dracaena elliptica Thumb

${ }^{*}$ Cordyline fruticosa (L.)

Goeppert.

SAN 138296

\section{AMARANTHACEAE}

*Amaranthus spinosus $\mathrm{L}$.

*Cyathula prostrata (L.)

SAN 138278

\section{ANACARDIACEAE}

Semecarpus cuneiformis Blanco.

\section{ANNONACEAE}

Desmos teijsmanii (Boert.) Merr.

SAN 138271

Phaeanthus ebrasteolatus

(Pers) Merr.

SAN 138288

Uvaria grandiflora Roxb. SAN 138270

\section{APOCYNACEAE}

Alstonia angustifolia Wall ex DC

SAN 138289

\section{ARACEAE}

*Acorus calamus L.

Aglaonema oblongifolium Schoot.

FRCSE 424

Alocasia macrorrhizos

(L.) G.Don f.

Homalomena propingna

Schoot.

FRCSE 414

\author{
Common \\ uses
}

\author{
Insasahi (Ka)
}

Stomach ach

Leaves

$\begin{array}{ll}\text { Sipak (Ka) } \quad \text { Tonic } \\ \text { Pipisokalaganan } & \text { Flatulence }\end{array}$

Leaves and Flowers

Root

(Ti)

$\begin{array}{lll}\text { Sansam Sau (P) } & \text { Epileptic seizures } & \text { Fresh leaves } \\ \text { Sansam Bawi (Ti) } & \text { Insect bites } \quad \text { Leaves }\end{array}$

Kutang

Wounds

Leaves

smoke to be inhaled

\begin{tabular}{|c|c|c|c|}
\hline $\begin{array}{l}\text { Molisun } \\
\text { Rumungkut (Ti) }\end{array}$ & Headache & Leaves & $\begin{array}{l}\text { Boiled in water } \\
\text { and as a bath }\end{array}$ \\
\hline Korokos (Ti) & Eyepain & Sap & $\begin{array}{l}\text { Applied directly } \\
\text { on eye }\end{array}$ \\
\hline Nolilitan (Ti) & $\begin{array}{l}\text { Waist pain and } \\
\text { stomach ache }\end{array}$ & Leaves & $\begin{array}{l}\text { Boiled in water to } \\
\text { make a tea }\end{array}$ \\
\hline $\begin{array}{l}\text { Tombirog (Ke) } \\
\text { Tambailik (Ti \& Ta) }\end{array}$ & Gastritis & Leaves & $\begin{array}{l}\text { Boiled in water to } \\
\text { make a tea }\end{array}$ \\
\hline Kusul (P) & $\begin{array}{l}\text { Stomach ache } \\
\& \text { fever }\end{array}$ & Rhizome & $\begin{array}{l}\text { Boiled in water to } \\
\text { make a tea }\end{array}$ \\
\hline Pilonos (Ta) & Boils & Leaves & $\begin{array}{l}\text { Pounded into paste } \\
\text { and applied on the } \\
\text { affected area }\end{array}$ \\
\hline Buntui (Ke) & Itchiness & $\begin{array}{l}\text { Sap from } \\
\text { the stem }\end{array}$ & $\begin{array}{l}\text { Applied directly to } \\
\text { the affected skin }\end{array}$ \\
\hline Nyato (Ta) & Feverish cold & Pith & $\begin{array}{l}\text { Heated and applied } \\
\text { on forehead }\end{array}$ \\
\hline
\end{tabular}

NOTE: *: introduced, naturalised or only known from cultivation; FRCSE: Forest Research Centre Sabah Ethnobotanical Collections Series; SAN: Sandakan Herbarium Series; P: Paluan; Ka: Kalabakan; Ke: Keningau; Ti: Timugon; Ta: Tagal. 


\section{specimen no. (if any) \\ ARISTOLOCHIACEAE \\ Aristolochia papillifolia}

Botanical name and voucher

Common

names (Dialect)

\author{
Medicinal
}

uses

Ding Hou.

FRCSE 412

Babas Lontong

(P\& Ta)

Antidote

jaundice,

inflammation of

liver (Paluon).

Diarrhoea (Tagal)

\section{BIGNONIACEAE}

Oroxylum indicum (L)

Kurz.

SAN 138284

Ulunan Sangku

Swelling

(Ti)

\section{BIXACEAE}

*Bixa orellana L. FRCSE 409

\section{BROMELIACEAE}

*Ananas comosus (L.)

Merr.

ingkauran $(P)$

Dandruff

Post partum treatment
(BI.) Merr
SAN 13823

Bunatol (Ti)

\section{COMBRETACEAE}

Combretum nigrescen

King

SAN 138290

Damat
Dumalarom (Ti)

Internal injury

COMPOSITAE

*Blumea balsamifera (L.)

DC.

*B. riparia (BI.) DC

FRCSE 407

Elephantopus tomentosus L.

FRCSE 419

*Synedrella nodiflora (L.)

Gaertn

FRCSE 418

\section{COSTACEAE}

Costus specious Koen.

FRCSE 416

\section{CUCURBITACEAE}

*Trichosanthes cucumerina L. SAN 138292
Tawawoh

( $P$ \& Ta)

Insasabu

(Ka \& Ta)

$$
\text { Hon }
$$

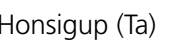

Sap from the young stem.

Leaves
Bark

Root

Sap from the stem

Root's bark

Young leaves

Method of Use

Boiled in water to make a tea

Soaked in hot water and rubbed on the effected area

Boiled in water to make a tea

Crushed and rubbed on the head

Stem is cut to get the sap and drink

Boiled in water to make a tea

Boiled in water to make a tea

Boiled in water to make a tea with a mixture of Hibiscus sp. (Malvaceae) FRCSE 408

Boiled in water to make a tea

Boiled in water and as a bath

Stem is cut to get the sap and drink

Pounded into paste and applied to the affected area 


\begin{tabular}{|c|c|c|c|c|}
\hline $\begin{array}{l}\text { Botanical name and voucher } \\
\text { specimen no. (if any) }\end{array}$ & $\begin{array}{l}\text { Common } \\
\text { names (Dialect) }\end{array}$ & $\begin{array}{l}\text { Medicinal } \\
\text { uses }\end{array}$ & Part Used & Method of Use \\
\hline \multicolumn{5}{|l|}{ DILLENIACEAE } \\
\hline $\begin{array}{l}\text { Dillenia grandifolia Wall. } \\
\text { Ex. Hk.f. et. Thom. }\end{array}$ & Dudungin $(\mathrm{Ta})$ & Stomach ache & Roots & $\begin{array}{l}\text { Boiled in water to } \\
\text { make a tea }\end{array}$ \\
\hline \multicolumn{5}{|l|}{ DIPTEROCARPACEAE } \\
\hline Shorea spp. (Eushorea) & Omnompik (Ta) & Food poisoning & Inner bark & $\begin{array}{l}\text { Pounded into } \\
\text { paste and eaten }\end{array}$ \\
\hline \multicolumn{5}{|l|}{ EUPHORBIACEAE } \\
\hline $\begin{array}{l}\text { Antidesma montanum BI. } \\
\text { SAN } 138297\end{array}$ & $\begin{array}{l}\text { Damat mandalom } \\
\text { (Ti) }\end{array}$ & Chest pain & $\begin{array}{l}\text { Root and } \\
\text { leaves }\end{array}$ & $\begin{array}{l}\text { Root is boiled in } \\
\text { water to make } \\
\text { a tea } \\
\text { Leaves are pounded } \\
\text { into paste and } \\
\text { applied on chest }\end{array}$ \\
\hline $\begin{array}{l}\text { Baccaurea lanceolata } \\
\text { (Miq.) Muell-Arg. }\end{array}$ & Limposu (P) & Abdomen pain & Fruit & $\begin{array}{l}\text { Pounded into } \\
\text { paste and applied } \\
\text { on the abdomen }\end{array}$ \\
\hline Bombax ceiba L. & Kapok $(P)$ & Vomitting blood & Bark & $\begin{array}{l}\text { Boiled in water to } \\
\text { make a tea }\end{array}$ \\
\hline $\begin{array}{l}\text { Bridellia stipularis (L) BI. } \\
\text { SAN } 138279\end{array}$ & Bolingkut (Ti) & $\begin{array}{l}\text { Diabetes Mellitus } \\
\text { Thrush }\end{array}$ & $\begin{array}{l}\text { Root } \\
\text { Fruit }\end{array}$ & $\begin{array}{l}\text { Pounded into paste } \\
\text { and applied on } \\
\text { the affected area }\end{array}$ \\
\hline Eupathorium odoratum L. & Lambaian (P) & Cuts and wounds & Leaves & $\begin{array}{l}\text { Pounded into paste } \\
\text { and applied on the } \\
\text { affected area }\end{array}$ \\
\hline $\begin{array}{l}\text { Glochidion macrostigma Hk.f. } \\
\text { SAN } 138273\end{array}$ & Sondot Laling (Ti) & Feverish cold & Leaves & $\begin{array}{l}\text { Boiled in water } \\
\text { and as a bath }\end{array}$ \\
\hline *Jatropha curcas L. & Jarak (Ke) & Gastritis & Leaves & $\begin{array}{l}\text { Boiled in water to } \\
\text { make a tea }\end{array}$ \\
\hline Macaranga gigentifolia Merr. & Binawong (Ka) & Thrush & $\begin{array}{l}\text { Sap from } \\
\text { the twig }\end{array}$ & $\begin{array}{l}\text { Cut the twig to get } \\
\text { the sap and applied } \\
\text { on the affected area }\end{array}$ \\
\hline $\begin{array}{l}\text { *Phyllanthus urinaria L. } \\
\text { SAN } 138282\end{array}$ & Pilujala (Ti) & Coughing & Entire plant & $\begin{array}{l}\text { Boiled in water to } \\
\text { make a tea }\end{array}$ \\
\hline
\end{tabular}

\section{FLACOURTIACEAE}

Casearia grewiaefolia Vent. var. grewiaefolia

Pounded into paste and applied on the affected area

\section{FLAGELLARIACEAE}

Flagellaria indica L. SAN 138300
Waau (Ti)

Pohodo (Ta)

\section{Semi-paralysis}

Entire plant

\section{GESNERIACEAE}

Cyrtandra aff. areolata (Staff.) Bl. Burtt.

FRCSE $428 \& 431$

Bloody stool Young stem
Skin disease

Boiled in water and as a bath

Raw stem is eaten and made a tea to treat bloody stool Ashes of burnt stem are applied to treat skin disease

NOTE: *: introduced, naturalised or only known from cultivation; FRCSE: Forest Research Centre Sabah Ethnobotanical Collections Series; SAN: Sandakan Herbarium Series; P: Paluan; Ka: Kalabakan; Ke: Keningau; Ti: Timugon; Ta: Tagal. 


$\begin{array}{llll}\begin{array}{l}\text { Botanical name and voucher } \\ \text { specimen no. (if any) }\end{array} & \begin{array}{l}\text { Common } \\ \text { names (Dialect) }\end{array} & \begin{array}{l}\text { Medicinal } \\ \text { uses }\end{array} & \text { Part Used }\end{array} \quad$ Method of Use

GRAMINEAE

*Eleusine indica (L.) Gaetner

Liagon $(P)$

Food poisoning

Entire plant

For food poisoning

Broken bones

Internodes

entire plant is

boiled to make tea

For broken bones

internodes are

crushed and applied

*Cymbopogon citratus

(DC.) Stapf.

Sohumau $(P) \quad$ Cough Leaves

Pounded into paste and applied on the affected area

*Imperata cylindrica

(L.) Rausch.

Lalang (Ka)

Bleeding

Young leaves

Pounded into paste and applied on the affected area

Zea mays $\mathrm{L}$.

Halai (Ka)

Stomach ache

Young fruit

Pounded into paste and applied on the stomach

\section{GUTTIFERAE}

* Garcinia mangostana L.

Timpurog (P)

Vomiting blood

Fruit

Eaten raw

\section{HYPOXIDACEAE}

Curculigo latifolia Dryander

Tambaka (Ta)

Wounds

Sap from

the pith of

the stem

Baing-Baing (Ti) Stomach ache

Young leaves

*Hyptis capitata Jack. SAN 138187

*Orthosiphon aristatus

(Blume) Miq.

Misai Kucing (Ta) Hypertension

Leaves

\section{LAURACEAE}

Lindera pipericarpa Boerl.

Laindos $(P)$

Litsea graciae Vidl.

FRCSE 420

L. odorifera Valeton

\section{LEGUMINOSAE}

Airgantha borneensis

(Oliv.) Brummit

SAN 138299

Cassia alata L.

Milletia nieuwenhuisii FRCSE 389

\begin{tabular}{|c|c|c|}
\hline Laindos (P) & $\begin{array}{l}\text { Antidote for } \\
\text { snake-bite }\end{array}$ & $\begin{array}{l}\text { Leaves, fruits } \\
\& \text { barks }\end{array}$ \\
\hline Novolo (P) & Joint dislocation $(\mathrm{P})$ & Inner barks \\
\hline Pengolaban ( $\mathrm{Ta}$ ) & Sprain (Tagal) & \\
\hline
\end{tabular}

Lawang $(\mathrm{P}) \quad$ Gastritis Bark

Stomach ache

Molisun High temperature Bark

Matamis (Ti) Toothache

Ringworm

Leaves

Ramus (Ka)

Thrush
Sap from the stem cut to get the sap and applied on the affected area

Pounded into paste and applied on the affected area

Boiled in water to make a tea

Pounded into paste and applied on the affected area

Pounded into paste and applied on the affected area

Pounded into paste and applied on the affected area

Pounded into paste and boiled in water to make a tea and as a gargle

Pounded into paste and applied on the affected area

Cut to get the sap and applied on the affected area 


$\begin{array}{llll}\begin{array}{l}\text { Botanical name and voucher } \\ \text { specimen no. (if any) }\end{array} & \begin{array}{l}\text { Common } \\ \text { names (Dialect) }\end{array} & \begin{array}{l}\text { Medicinal } \\ \text { uses }\end{array} & \text { Part Used }\end{array} \quad$ Method of Use

\section{LEGUMINOSAE cont.}

\begin{tabular}{|c|c|c|c|c|}
\hline *Mimosa pudica L. & $\begin{array}{l}\text { Sikot Mou (Ke) } \\
\text { Tenom Molu (Ka) }\end{array}$ & Stomach ache & Root & $\begin{array}{l}\text { Boiled in water to } \\
\text { make a tea }\end{array}$ \\
\hline Parkia singularis Miq. & Kundai $(P)$ & Kidney cleanser & Fruit & Eaten raw \\
\hline $\begin{array}{l}\text { Spatholobus cf. gyrocarpus } \\
\text { Benth. in Miq. } \\
\text { FRCSE } 410\end{array}$ & Ramus (Ka) & Thrush & $\begin{array}{l}\text { Sap from } \\
\text { the stem }\end{array}$ & $\begin{array}{l}\text { Cut to get the sap } \\
\text { and applied on } \\
\text { the mouth }\end{array}$ \\
\hline \multicolumn{5}{|l|}{ MALVACEAE } \\
\hline \multicolumn{5}{|l|}{ *Urena lobata L.I } \\
\hline \multirow[t]{2}{*}{ SAN 138286} & Injilokot (Ti) & Thrush & Bark & $\begin{array}{l}\text { Burnt to ashes } \\
\text { and applied }\end{array}$ \\
\hline & & $\begin{array}{l}\text { Bowel movement } \\
\text { inducer }\end{array}$ & Flowers & $\begin{array}{l}\text { Pounded into paste } \\
\text { and applied on } \\
\text { the abdomen }\end{array}$ \\
\hline * Sida rhombifolia L. & Dalupang $(P)$ & Antidote & Leaves & $\begin{array}{l}\text { Boiled in water to } \\
\text { make a tea }\end{array}$ \\
\hline \multicolumn{5}{|l|}{ MELIACEAE } \\
\hline $\begin{array}{l}\text { Lansium domesticum } \\
\text { Corr. Serr. }\end{array}$ & Langsat (Ka \& P) & $\begin{array}{l}\text { Stomach ache } \\
\text { Diarrhoea } \\
\text { Colic }\end{array}$ & Bark & $\begin{array}{l}\text { Pounded into paste } \\
\text { and applied on } \\
\text { the abdomen }\end{array}$ \\
\hline \multicolumn{5}{|l|}{ MENISPERMACEAE } \\
\hline $\begin{array}{l}\text { Coscinium fenestratum } \\
\text { (Gaerth.) Cole } \\
\text { FRCSE } 433\end{array}$ & $\begin{array}{l}\text { Babas Lingungan } \\
(\mathrm{Ta})\end{array}$ & $\begin{array}{l}\text { Thinning and } \\
\text { yellowing skin }\end{array}$ & Entire plant & $\begin{array}{l}\text { Boiled in water } \\
\text { to make a tea }\end{array}$ \\
\hline Fibraruea tinctoria Lour. & Tolungon ( $\mathrm{P} \& \mathrm{Ta})$ & $\begin{array}{l}\text { Anti-malarial } \\
\text { (Tagal) } \\
\text { Hypertension }\end{array}$ & Stem & $\begin{array}{l}\text { Boiled in water to } \\
\text { make a tea }\end{array}$ \\
\hline $\begin{array}{l}\text { Stephania corymbosa } \\
\text { FRCSE } 396\end{array}$ & $-(\mathrm{Ka})$ & Antidote & Stem & $\begin{array}{l}\text { Boiled in water to } \\
\text { make a tea }\end{array}$ \\
\hline $\begin{array}{l}\text { Tinospora crispa (L.) } \\
\text { Hook.f. \& Thomsen }\end{array}$ & $-(\mathrm{Ka})$ & Anti-malarial & Stem & $\begin{array}{l}\text { Boiled in water to } \\
\text { make a tea }\end{array}$ \\
\hline \multicolumn{5}{|l|}{ RUBIACEAE } \\
\hline $\begin{array}{l}\text { *Jasminum aculeatum } \\
\text { (Blanco.) Merr. }\end{array}$ & $\begin{array}{l}\text { Onsom-onsom } \\
\text { (Ti) }\end{array}$ & Flatulence & Leaves & $\begin{array}{l}\text { Pounded into paste } \\
\text { with warm water } \\
\text { and applied on the } \\
\text { affected area }\end{array}$ \\
\hline $\begin{array}{l}\text { *Psychotria cf. } \\
\text { sarmentosa Bl. }\end{array}$ & Solovondo (Ta) & Itchiness & Stem & $\begin{array}{l}\text { Burnt to ashes and } \\
\text { rubbed on the } \\
\text { affected area }\end{array}$ \\
\hline \multicolumn{5}{|l|}{ ZINGIBERACEAE } \\
\hline *Curcuma longa L. & Kunyit (Ta) & Anti-fungal & Rhizome & $\begin{array}{l}\text { Pounded into paste } \\
\text { and applied on the } \\
\text { affected area }\end{array}$ \\
\hline *Zingiber officinale Roscoe & Halia (Ta) & Flatulence & Rhizome & $\begin{array}{l}\text { Pounded into paste } \\
\text { and applied on the } \\
\text { affected area }\end{array}$ \\
\hline
\end{tabular}

NOTE: *: introduced, naturalised or only known from cultivation; FRCSE: Forest Research Centre Sabah Ethnobotanical Collections Series; SAN: Sandakan Herbarium Series; P: Paluan; Ka: Kalabakan; Ke: Keningau; Ti: Timugon; Ta: Tagal. 
TABLE 2. OTHER USEFUL PLANTS USED BY THE MURUTS IN SABAH, MALAYSIA.

$\begin{array}{llll}\begin{array}{l}\text { Botanical name and voucher } \\ \text { specimen no. (if any) }\end{array} & \begin{array}{l}\text { Common } \\ \text { names (Dialect) }\end{array} & \text { Category } & \text { Part Used }\end{array} \quad$ Method of Use

ARACEAE

Homalomena FRCSE 414

Fish poison

Pith

\section{ANACARDIACEAE}

Mangifera indica L.

M. pajang Kost.

Longgom (Ka) Edible fruit
Bambangan (Ka) "

ANNONACEAE

Gonoithalamus woodii Merr. FRCSE $390 \& 415$

Tampaliu (Ka) Kuluor (Ta)

Ghost scare

\section{BAMBUSOIDEAE}

*Bambusa blumeana Schult.

Gigantochloa levis

Kayawen(Ti)

* Schizostachyum brachycladum Bulu (Ti) Kurz.

\section{S. pilosum S. Dransf.}

Pus (Ka)

BOMBACACEAE

Durio graveolens Becc.

Ruyan (Ka)

D. zibethinus Murray

Lampun (Ka)

\section{BROMELIACEAE}

Ananas comosus (L) Merr.

Tingkauran (P)

Cotton

Silinggaun (Ka)

Ornamental

Bunatol (Ti)

Poikilospermum suaveolens

(BI.) Merr.

\section{DIPTEROCARPACEAE}

Shorea parvistipulata Heim. FRCSE 391

Roloi (Ka)

Stimulant

\section{EUPHORBIACEAE}

Baccaurea puberula Merr.

Tampoi kuning (Ka)

B. lanceolata (Miq.) Muell-Arg. Lipasu (Ka)

Bombax ceiba L.

Kapok (P)

*Manihot esculenta Crantz.
Pillow

Edible fruit

Edible

(n)

Mesocarp

$"$

Whole plant

on the upper main entrance or burnt

Entire

Young leaves

pepper leaf as substitute for gambir

Pith is pounded and soaked in water/stream

Eaten ripe, or unripe as pickle

Eaten ripe

Cooked

Cooked

Cut

Cut

Cut small

Eaten ripe

Matured leaves are beaten and woven

Bug tree

Cooked

Ripe

Aril \& $\quad$ Ripe aril

Mesocarp Mesocarp eaten as pickle

Matured fruit Fibre for pillows

Root (tuber) Cook

Leaves 


\begin{tabular}{|c|c|c|c|c|}
\hline $\begin{array}{l}\text { Botanical name and voucher } \\
\text { specimen no. (if any) }\end{array}$ & $\begin{array}{l}\text { Common } \\
\text { names (Dialect) }\end{array}$ & Category & Part Used & Method of Use \\
\hline \multicolumn{5}{|l|}{ EUPHORBIACEAE cont. } \\
\hline Koilodepas longifolium Hook. f. & Kayu Ulas (Ka) & Bow & Bole & Smaller bole \\
\hline $\begin{array}{l}\text { Macaranga tanarius (L) } \\
\text { Muell. Arg. }\end{array}$ & $\begin{array}{l}\text { Daun Bayangan } \\
(\mathrm{Ka})\end{array}$ & Wrapper & Leaves & To wrap rice \\
\hline \multicolumn{5}{|l|}{ HYPOXIDACEAE } \\
\hline \multicolumn{5}{|l|}{ Curculigo latifolia Dryander. } \\
\hline FRCSE 413 & Tambaka (Ta) & Edible fuit & Aril & Ripe \\
\hline \multicolumn{5}{|l|}{ GRAMINEAE } \\
\hline *Saccharum officianarum L. & Tebu (Ka) & Drinkable & Sap & Matured stem \\
\hline *Zea mays L. & Halai (Ka) & Edible fruit & Cotyledons & Cook \\
\hline \multicolumn{5}{|l|}{ GUTTIFERAE } \\
\hline Garcinia parvifolia (Miq.) Miq. & Kandis (Ka) & Edible fruit & $\begin{array}{l}\text { Aril \& } \\
\text { Mesocarp }\end{array}$ & $\begin{array}{l}\text { Ripe aril } \\
\text { Ripe mesocarp } \\
\text { dried and cooked } \\
\text { as vegetable }\end{array}$ \\
\hline \multicolumn{5}{|l|}{ FLACOURTIACEAE } \\
\hline $\begin{array}{l}\text { Trichadenia philippensis Merr } \\
\text { FRCSE } 421\end{array}$ & Tulok-Ulok (Ta) & Magical use & Leaves & $\begin{array}{l}\text { To make thief's feet } \\
\text { bleed; leaves are } \\
\text { placed on the sole }\end{array}$ \\
\hline \multicolumn{5}{|l|}{ LAURACEAE } \\
\hline Lindera pipericarpa Boerl. & Laindos $(P)$ & Lethal to chicken & Fruits & Ripe \\
\hline $\begin{array}{l}\text { Litsea graciae Vidl. } \\
\text { FRCSE } 420\end{array}$ & $\begin{array}{l}\text { Novolo (P) } \\
\text { Pengoloban (Ta) }\end{array}$ & Edible fruit & Aril & Ripe \\
\hline Eusideroxylon zwageri T. \& B. & Belian (Ka) & Blow-pipe & Young bole & $\begin{array}{l}\text { A hole made } \\
\text { through }\end{array}$ \\
\hline \multicolumn{5}{|l|}{ LEGUMINOSAE } \\
\hline $\begin{array}{l}\text { Bauhinia kockiana Korth. } \\
\text { Var. kockiana } \\
\text { FRCSE } 414\end{array}$ & Kulih Bakah (Ta) & $\begin{array}{l}\text { Prevent evil-craft } \\
\text { from enemy }\end{array}$ & Entire plant & Fresh \\
\hline $\begin{array}{l}\text { Derris cf. trifolia Lour. } \\
\text { FRCSE } 388\end{array}$ & Apa (Ka) & Flavour & Leaves & Cooked \\
\hline Milletia nieuwenhuisii J.J. Smith & Romus (Ta) & Knife handle & Wood & - \\
\hline \multicolumn{5}{|l|}{ MARANTACEAE } \\
\hline $\begin{array}{l}\text { Donax canniformis (Forst.) } \\
\text { Schum }\end{array}$ & $\operatorname{Lias}(\mathrm{Ta})$ & Basketwork & $\begin{array}{l}\text { Outer stem } \\
\& \text { fish trap }\end{array}$ & $\begin{array}{l}\text { Stem split into } \\
\text { strips }\end{array}$ \\
\hline \multicolumn{5}{|l|}{ MELASTOMATACEAE } \\
\hline $\begin{array}{l}\text { Dissochaeta monticula BI. } \\
\text { FRCSE } 427\end{array}$ & Bina (Ta) & $\begin{array}{l}\text { Lethal to } \\
\text { mammals }\end{array}$ & Root's sap & $\begin{array}{l}\text { Used as blowpipe } \\
\text { poison }\end{array}$ \\
\hline \multicolumn{5}{|l|}{ MELIACEAE } \\
\hline Lansium domesticum Corr. Serr. & Langsat & Edible fruit & Aril & Ripe \\
\hline
\end{tabular}

NOTE: *: introduced, naturalised or only known from cultivation; FRCSE: Forest Research Centre Sabah Ethnobotanical Collections Series; SAN: Sandakan Herbarium Series; P: Paluan; Ka: Kalabakan; Ke: Keningau; Ti: Timugon; Ta: Tagal. 


\section{specimen no. (if any) \\ MENISPERMACEAE}

Botanical name and voucher

Common names (Dialect)

Pycnarrhena of.

tumetacta Miers.

FRCSE 388

\section{MUSACEAE}

Musa textiles Nee.

Apa $(\mathrm{Ka})$

Flavour

Leaves

Part Used

Method of Use

Category

Part Used

\author{
Punti (Ka)
}

Edible fuit
Vegetable

$\begin{array}{ll}\text { Aril } & \text { Ripe } \\ \text { Pith \& un- } & \text { Cook } \\ \text { opened flowers } & \end{array}$

\section{MORACEAE}

Antiaris toxicaria (Pers.) Lesch.

Artocarpus communis

J.R. \& G. Foster

Paliu (Ka)

Poison

Latex

Poisons darts

A. elasticus Bl.

A. odoratissimus Blanco.

A. integer (Thumb.) Merr.

A. tamaran Becc.

Ficus beccarii King.

FRCSE 417

\section{MYRTACEAE}

*Psidium guajava L.

\section{MYRSINACEAE}

Embelia philippinensis A. DC. FRCSE 387

\section{PALMAE}

Areca catechu L.

Arenga undulitifolia Becc.

Calamus spp.

*Cocos nucifera L. Becc.

Cryostachys lakka Becc.

Eugissonia utilis Becc. FRCSE 393

Licuala spp.

Meteroxylon sagu Rottb.

Oncosperma tigillarium (Jack) Ridl
Kaliabas (P) Liabas (Ka)

\section{Kemansi (Ka)}

Edible fruit

Aril

Ripe

Puputul (P)

Bird trap

Tarap (Ka)

$"$

Luon (Ka)

Tatali (Ta)

Papaling (Ka)

Edible

Young leaves

Salad

\begin{tabular}{|c|c|c|c|}
\hline $\begin{array}{l}\text { Kusob (Ta) } \\
\text { Pinang (Ka) }\end{array}$ & Stimulant & Endocarp & $\begin{array}{l}\text { Mature fruit } \\
\text { eaten raw }\end{array}$ \\
\hline Polod (Ta) & Fire starter (tinder) & Soft net & - \\
\hline $\begin{array}{l}\text { Rotan lasun, } \\
\text { pipit (Ka) }\end{array}$ & $\begin{array}{l}\text { Vegetable } \\
\text { Rope }\end{array}$ & $\begin{array}{l}\text { Tip of the plant } \\
\text { Cane }\end{array}$ & $\begin{array}{l}\text { Cook } \\
\text { Matured cane }\end{array}$ \\
\hline Piasau (Ka) & $\begin{array}{l}\text { Edible fruit } \\
\text { Broom }\end{array}$ & $\begin{array}{l}\text { Endocarp/Juice } \\
\text { Outer part of } \\
\text { old fruit. }\end{array}$ & $\begin{array}{l}\text { Young/matured } \\
\text { Matured endocarp } \\
\text { used as source } \\
\text { of oil }\end{array}$ \\
\hline
\end{tabular}

Paluon (Ka)

Edible Sago

Pith

Pressed Dart's cock Pith Matured pith

Silan/Tanom (Ta) Vegetable

Pith

Cooked

Lumbio (Ta)

Edible sago

Nibong (Ka)

Vegetable
Pressed

Raw or cook 


\begin{tabular}{|c|c|c|c|c|}
\hline $\begin{array}{l}\text { Botanical name and voucher } \\
\text { specimen no. (if any) }\end{array}$ & $\begin{array}{l}\text { Common } \\
\text { names (Dialect) }\end{array}$ & Category & Part Used & Method of Use \\
\hline \multicolumn{5}{|l|}{ PALMAE cont. } \\
\hline $\begin{array}{l}\text { Pandanus leuconatus } \\
\text { B.C. Stone } \\
\text { FRCSE } 395\end{array}$ & Boboungis (Ka) & Matting & Leaves & Dried and woven \\
\hline $\begin{array}{l}\text { Plectocomiopsis geminiflora } \\
\text { (Griff.) Becc. } \\
\text { FRCSE } 411\end{array}$ & Ambarua (Ta) & Vegetable & Stem's shoot & Cooked \\
\hline \multicolumn{5}{|l|}{ SAPINDACEAE } \\
\hline $\begin{array}{l}\text { Lepisanthes fruticosa } \\
\text { (Roxb.) Leenh. } \\
\text { SAN } 138272\end{array}$ & Talikasan (Ti) & Edible fruit & Aril & Ripe \\
\hline Nephelium lappaceum L. & Kayakan (Ka) & $"$ & $"$ & $"$ \\
\hline $\begin{array}{l}\text { N. ramboutan-ake } \\
\text { (Labill.) Leenh. }\end{array}$ & Rumokot (Ka) & $"$ & " & $"$ \\
\hline \multicolumn{5}{|l|}{ SOLANACEAE } \\
\hline Solanum turvom Sw. & Lintahun $(\mathrm{Ta})$ & Softener & Fruit & Cooked with meat \\
\hline \multicolumn{5}{|l|}{ FUNGI } \\
\hline $\begin{array}{l}\text { Polyporus umbellatus } \\
\text { FRCSE } 392\end{array}$ & Ongkulan (Ka) & Edible mushroom & - & Cooked \\
\hline $\begin{array}{l}\text { Auricularia auricula-judae } \\
\text { FRCSE } 394\end{array}$ & Tondungol (Ka) & $"$ & $"$ & $"$ \\
\hline Cantharellus cibarius & Sunsulit (Ka) & " & $"$ & $"$ \\
\hline \multicolumn{5}{|l|}{ FERN } \\
\hline $\begin{array}{l}\text { Nephrolepis biserrata } \\
\text { (Sw.) Schoot. }\end{array}$ & Pakis (Ta) & Vegetable & Tip & Cooked \\
\hline
\end{tabular}

NOTE: *: introduced, naturalised or only known from cultivation; FRCSE: Forest Research Centre Sabah Ethnobotanical Collections Series; SAN: Sandakan Herbarium Series; P: Paluan; Ka: Kalabakan; Ke: Keningau; Ti: Timugon; Ta: Tagal.

In most of the treatments with medicinal plants, the herbal preparations are administered orally. The specific amount of material could not be ascertained. The recommended dosage is normally a handful of leaves or few pieces of root, rhizome, stem or bark, flowers, fruits and seeds. For small herbs, the whole plant or a few plants may be used. Leaves are the most common part used. The roots are the second-most common part used in traditional medicine, followed by bark and the reproductive parts. They are used fresh or dry, chewed, or boiled in water and the decoction taken as tea. The herbal preparation is usually taken once or twice daily until the patient recovers. The ailments described by the plant informants have been interpreted according to Pescar and Nelson (1996). 


\section{Discussion}

The knowledge of medicinal plants in Murut communities is normally passed on from generation to generation but this practice seems to be vanishing. It was estimated that there are only two to three persons in any village who know the many uses of plants, and they were usually the elderly. When asked if they still relied on herbal treatment, the response was that not all of the medicinal plants are used nowadays. Some of the reasons given were:

Only the elderly know most of the plants and where to get them: Some of the very useful medicinal plants are only known by the elderly, but because they are very old and their vision is mostly now poor this makes it difficult for them to recognize the plants. Even if their vision is good they may not be able to walk far.

Preparation of herbs takes time including finding the plants in the forest: The process of looking for the plants in the forest and the preparation of remedies is very laborious compared to buying them at the nearby market or going to the hospital. Each of the districts has its own government district hospital except for Kalabakan district where a government clinic is provided. These modern facilities make rural people dependent on ready-made modern medicines.

Religion: Some religions do not allow the use of any biological material for medicines.

Lost of interest of younger generations: Ancient customs of plant utilization are gradually disappearing. The younger generation is generally unable to recognise the plants nor their traditional use. They are dependent on modern medicines.

Migration to urban areas: Many Muruts people have migrated to urban areas to find paid work and access modern facilities.

Opening of forests: In recent years, Sabah faced a very rapid opening of forest for timber harvesting, development and agriculture. This has caused enormous changes to occur in Murut lives and in the way they utilize plant resources.

Realizing the economic potential of these medicinal and other useful plants and to conserve them, activities at Federal as well as at State level have been launched to save these plants from being neglected. At a Federal level, the Ministry of Education has introduced co-curriculum activities such as establishing a medicinal herb garden. School children at primary and secondary level are encouraged to learn about the medicinal value of some plants, for example, Eurycoma longifolia or 'Tongkat Ali' and Morinda citrifolia or 'Mengkudu'. The Ministry of Health Malaysia has also conducted a nation-wide campaign on the use and conservation of traditional medicinal plants. To date the Ministry has already registered about 1,300 medicinal plant products that are being sold in the markets. At the State level, the Sabah government has passed a new law this year [2001] concerning the use of indigenous plants; it is called the Sabah Biodiversity Enactment. The purpose is to regulate the studies of useful plants such as medicinal plants and for conservation of natural resources. The Chief Department of Sabah established a working group comprising all State departments, agencies and NGOs called the Sabah Herbal Industry Committee early this year [2001]. The objectives are to act as the Secretariat at State level to systematically document and regulate the utilization of the indigenous medicinal plants in a sustainable manner. 


\section{Conclusions}

From this survey we have documented a total of 91 species of plants that are being used by Muruts throughout Sabah. Sixty-eight species are medicinal plants and 64 species are other useful plants, which includes wild fruit, handicraft materials, plants for social and religious purposes, and poisons. These plants are available in direct vicinity of the village or in the nearby forest.

The Muruts have a rich ethnobotanical knowledge, but this is fading due to migration to urban areas, a loss of interest among the young, religious restrictions or dependence on modern medicine. Opening of forest areas for agriculture, development and timber harvesting have also made the resources scarce.

The wealth of Murut knowledge of medicinal and other useful plants points to a great potential for research and the discovery of new drugs to fight diseases, new foods and other new uses.

\section{Acknowledgments}

I would like to acknowledge the State government of Sabah especially the Forestry Department Sabah for the support that I received throughout the surveys and for granting me approval to present this paper in Sydney. During the surveys Research Assistants Mr. George Majawat, Mr. Baraham Buhari, Mr. Lajiman Wasai have helped me and I would like to thank them for their continued support. I would like to thank retired Assistant Research Officer Mr. Leopold Madani for helping on the identification of specimens. This work wouldn't be possible if not for the participation by all Muruts villagers throughout Sabah. This paper is dedicated to their community in the hope that it will contribute in some small way towards their future well-being. I would like also to acknowledge the contributions of Sabah Museum, Sabah Archives and Tenom Murut Cultural Centre for being very helpful providing Muruts ethnography information. Last but not least I would like to thank the $5^{\text {th }}$ Flora Malesiana Symposium Committee in Sydney for accepting my paper.

\section{References}

Department of Statistics, Malaysia (2001). Monthly Statistical Bulletin Sabah. Jun. (Sabah Branch). Fasihuddin, B.A. and Hasmah, R. (1992). Medicinal plants of the Murut community in Sabah. Pp 460-467. In Ghazzaly, I., Siraj, O. and Murtedza, M. (eds). Forest Biology and Conservation in Borneo. (Centre for Borneo Studies: Kota Kinabalu, Sabah).

Guntavid, J.P. (1992). Traditional medicinal plants: their uses by the Tengala Muruts and Dusun/Kadazan of Tambunan. A paper presented in Borneo Research Council $2^{\text {nd }}$ Biennial International Conference, Kota Kinabalu, Sabah. (Borneo research Council).

King, J. (1992). A Preliminary Update to the Language Situation in Sabah. Summer Institute of Linguistics.

Martin, G. (1995). Ethnobotany. A 'People and Plants' Conservation Manual. (WWF International, UNESCO, Royal Botanic Gardens, Kew, UK, Chapman \& Hall).

Pescar, S.C. and Nelson, C.A. (1996). The Wordsworth Medical Companion: A guide to symptoms and illnesses. (Wordsworth Reference, Mackays, Great Britain).

Tombung, R. (1991). Keluarga Dusun. (Persatuan Dusun Sabah Bersatu: Kota Kinabalu). 
Kulip, Ethnobotany of Muruts, Sabah

\section{APPENDIX 1: LIST OF MEDICINAL PLANTS OF MURUTS}

Botanical Name

Acorus calamus L. ${ }^{3}$

Aglaia rivularis Merr. ${ }^{1}$

Aglaonema oblongofolium Schoot. *

Agave cf. weberi ${ }^{3}$

Airgantha borneensis (Oliv.) Brummit. ${ }^{3}$

Alocasia macrorrhizos*

Aloe vera L. ${ }^{2}$

Alstonia angustifolia Wall ex $\mathrm{DC}^{3}$

Amaranthus spinosus L. ${ }^{3}$

Ananas comosus (L.) Merr. *

Annona muricata L. ${ }^{2}$

Antidesma montanum Bl. ${ }^{2}$

Areca catechu L. ${ }^{2}$

Arenga undulitafolia Becc. ${ }^{3}$

Aristolochia papillifolia Ding Hou. ${ }^{3}$

Artocarpus elasticus Reinw ex Bl. ${ }^{2}$

Artocarpus tamaran Becc. ${ }^{3}$

Baccaurea lanceolata (Miq.) Muell-Arg. ${ }^{3}$

Bauhinia sp. ${ }^{2}$

B. semibifida Roxb. ${ }^{1}$

Begonia sp. ${ }^{1}$

Bixa orellana L. ${ }^{3}$

Blechnum orientale L. *

Blumea balsamifera Harn. *

B. riparia $(\mathrm{BI}) \mathrm{DC}^{3}$

Bombax ceiba L. ${ }^{3}$

Bridella stipularis (L) Bl. ${ }^{3}$

Caesalpinia sappan $\mathrm{L}^{2}$

Calamus pogonacanthus Becc. Ex. K. Winkl. ${ }^{1}$

Canarus sp. ${ }^{2}$

Capsicum frutescens $\mathrm{L}^{2}$

Casearia grewiaefolia Vent. Var. grewiaefolia ${ }^{3}$

Cassia alata L. *

Centotheca lappacea (Linn.) Desv. ${ }^{1}$

Centella asiatica (L.) Urban*

Clausena excavata Burm. ${ }^{1}$

Colocasia antiquorum Schott. ${ }^{1}$

Combretum nigrescens King ${ }^{3}$

Cocos nucifera L. *
Murut Name

Kusul (P)

Kalambiau

Pilonos (Ta)

Sipak (Ka)

Molisun Matamis (Ti)

Buntui (Ke)

Bunga Raja

Tambailik (Ti \& Ta)

Tombirog (Ke)

Sansam Sau (P)

Tingkauran $(P)$

Lampun Belanda

Damat Mandalom (Ti)

Kusauh

Polod (Ta)

Babas Lontong (Pa \& Ta)

Kikian

$-$

Limposu (Pa)

Pilasang

Babaya Songkulibang

Pamamampang

Puloh (Ta)

Paku

Tawawoh (P \& Ta)

Kapok (Pa)

Bolingkut (Ti)

Sapang

Sambunil

Dalat

Baras

Salokdan (Ti)

Balilang (Pa), Emon (Ke)

Pampopoi

Pegaga

Tataih Nu Manuk

Ungkubab Nu Labi

Damat Durnalarom (Ti)

Piasau (Ta)
Collection No.

FRCSE 424

SAN 138299

SAN 138289

SAN 138297

FRCSE 412

FRCSE 409

FRCSE 407

SAN 138279

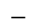

$-$

SAN 138276

$-$

SAN 138290

$\mathrm{P}=$ Paluan, $\mathrm{Ka}=$ Kalabakan, $\mathrm{Ke}=$ Keningau, $\mathrm{Ti}=$ Timugon and $\mathrm{Ta}=$ Tagal

$1=$ Collected by Guntavid (1992) $\quad 2$ = Fasihuddin \& Hasmah (1992)

$3=$ Collected by Kulip (2001) * $\quad$ = Collected by all authors 


\section{Botanical Name}

Cordyline fruticosa (L.) Goeppert ${ }^{3}$

Coscinium fenestratum (Gaerth.) Colebr. ${ }^{3}$

Costus specious Koen.*

Curculigo latifolia Dryander*

Curcuma domestica Val. ${ }^{3}$

Cyathula prostrata (L) BI. ${ }^{3}$

Cymbopogon citratus (Nees.) Stapf. *

Cyrtandra aff. areolata (Staff.)BI Burtt. ${ }^{3}$

Dendrobium umbellatom Reichb.f. ${ }^{2}$

Desmos teijsmannii (Boert.)Merr. ${ }^{3}$

Dillenia grandifolia Wall.ex. Hk.f. et. Thorn²

Dillenia sp. ${ }^{2}$

Dinochloa sp. ${ }^{2}$

D. scabrida S. Dransf. ${ }^{2}$

Dracaena umbratica Ridl. ${ }^{1}$

Elephantopus scaber L. ${ }^{2}$

E. tomentosus L. ${ }^{3}$

Eleusine indica (L) Gaetner.*

Erechtites valerianaefolia L. ${ }^{2}$

Eupathorium odoratum L.*

Eurycoma longifolia Jack.*

Fibraruea tinctoria Lour. ${ }^{3}$

Ficus aurantiacea Griff. ${ }^{2}$

F. deltoidea Jack. ${ }^{2}$

F. lepicarpa Bl. ${ }^{3}$

F. racemosa L. var elongata ${ }^{3}$

Ficus sp. ${ }^{2}$

Flagellaria indica L. ${ }^{3}$

Forrestia griffithii C.B. Clarke

Garcinia mangostana L. ${ }^{3}$

Glochidion macrostigma Hk.f.?

Graptophyllum pictum (L.) Griffithi

Hanguana malayana Jack. ${ }^{1}$

Helecia serrata (R.Br.) BI. ${ }^{3}$

Hibsicus rosa-sinensis L. ${ }^{2}$

Hibiscus sp. ${ }^{3}$

Homalium foetida (Roxb.) Benth ${ }^{1}$

Homalomena propingna Schoot. ${ }^{3}$

Homalanthus populneus Geisel. ${ }^{2}$

Hoya sp. ${ }^{1}$

Hydnophtyum formicarium Jack $^{3}$

Hypoestes sp. ${ }^{2}$

Hypolytrum nemorum (Vahl) Spreng ${ }^{1}$

\section{Murut Name}

Pipisokalaganan (Ti)

Babas Lingungan (Ta)

Insasabu (Ka \& Ta)

Tambaka (Ta)

Samsam Bawi (Ti)

Sohumau (Pa)

Pohodo (Ta)

Tingasu

Molisun Rumungkut

Tembakau (Ka)

Baran

"

Dolol Apui

Lambrunai

Honsigup ( $\mathrm{Ta}$ )

Liagon (Pa)

Sumayon

Lambaian $(\mathrm{Pa})$

Duli (P) Ruli (Ta)

Tolungon ( $\mathrm{P} \& \mathrm{Ta}$ )

Silabon-rondoh

Agolauran

Lintotobow (Ke)

Tandilan (P)

Mamponoh

Waau (Ti)

Tatapis Da Aputulan

Timpurog (Pa)

Sondot Laling (Ti)

Lalamih

Tatapis Da Umbir

Andaun Motukal (Ti)

Bunga Raya

Lulumada

Nyato $(\mathrm{Ta})$

Sipapaloi

Pongkukubab

Musalag Noh Kilou (P)

Matopait

Balasan Sungei

\section{Collection No.}

SAN 138296

FRCSE 433

FRCSE 416

FRCSE 413

SAN 138278

FRCSE 428 \& 431

SAN 138271

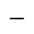

-

FRCSE 419

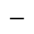

$-$

SAN 138300

SAN 138273

SAN 138291

FRCSE 408

FRCSE 414

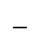

-

-

-

\footnotetext{
$\mathrm{P}=$ Paluan, $\mathrm{Ka}=$ Kalabakan, $\mathrm{Ke}=$ Keningau, $\mathrm{Ti}=$ Timugon and $\mathrm{Ta}=$ Tagal

$1=$ Collected by Guntavid (1992) $\quad 2$ = Fasihuddin \& Hasmah (1992)

$3=$ Collected by Kulip (2001) * $\quad$ Collected by all authors
} 


\section{Botanical Name}

Hyptis capitata Jack. ${ }^{3}$

Imperata cylindrica (L) Rausch. *

Ixora capillaries Boern. ${ }^{3}$

Jasminum aculeatum (Blanco) Merr. ${ }^{3}$

Jatropha curcas L. ${ }^{3}$

Justicia gendarussa L. ${ }^{3}$

Kalanchoe pinnata (Lam.) Pers. *

Lansium domesticum Corr. Serr. ${ }^{3}$

Leucosyke capitella (Poir.) Wedd.*

Lindera pipericarpa Boerl. ${ }^{3}$

Litsea graciae Vidl. ${ }^{3}$

L. odorifera Valeton ${ }^{3}$

Macaranga gigentifolia Merr. ${ }^{3}$

Micromelum minatum (Frost.) Seem ${ }^{3}$

Millettia nieuwenhuisii J.J. Smith ${ }^{3}$

Mimosa pudica L. *

Neonauclea calycina Merr. ${ }^{3}$

Oroxylum indicum (L) Vent. ${ }^{3}$

Orthosiphon aristatus (Blume) Miq. ${ }^{3}$

Parashorea malaanonan Merr. ${ }^{1}$

Parkia singularis Miq. ${ }^{3}$

Paspalum conjugatum Berg. ${ }^{2}$

Pavetta sp. ${ }^{1}$

Pedilanthus tithymaloides Poit.*

Phaeanthus ebrasteleolatus (Pres) Merr ${ }^{3}$

Phyllanthus urinaria L. ${ }^{3}$

Piper betle L. *

Piper carinum BI.*

Plectocomiopsis geminiflora (Griff.) Becc. ${ }^{3}$

Plumeria rubra L. ${ }^{1}$

Poikilospermum suaveolens (BI.) Merr. ${ }^{3}$

Polyalthia sp. ${ }^{1}$

P. bullata King 2

Praravinia suberosa Merr. ${ }^{1}$

Pronephrium asperum (Prest.) $\mathrm{Holtt}^{2}$

Psidium guajava L. *

Psychotria of. sammontosa Bl. ${ }^{3}$

Saurauia longistyla Merr. ${ }^{1}$

Selaginella argentea Sym. ${ }^{2}$

Semecarpus cuneiformis Blanco. ${ }^{3}$

Schismatoglottis sp. ${ }^{1}$

Schindapsus perakensis Hook.F. ${ }^{1}$

Scleria bancana Miq. ${ }^{1}$

Shorea spp. ${ }^{3}$ (Eushorea group)

\section{Murut Name}

Baing-baing (Ti)

Lalang (Ka)

Angin Tolunsung (Ti)

Onsom-Onsom (Ti)

Jarak (Ke)

Insasahi (Ka)

Tanom Tombiog

Langsat (Ka \& P)

Balawian

Laindos (P)

Novolo $(P)$,

Pengolaban (Ta)

Lawang $(P)$

Binawong ( $\mathrm{Ka}$ )

Kimamansak (Ka)

Ramus (Ka)

Sikot Mou (Ke),

Tanom Molu (Ka)

Kembalu (P)

Ulunan Sangku (Ti)

Misai Kucing (Ta)

Melapi

Kundai $(P)$

Belandak

Buntungon

Tatapis Tindukon

Korokos (Ti)

Pilujala (Ti)

Molur Malat (Ti)

Kimput-Pilot (Ta)

Ambarua (Ta)

Campaka

Bunatol (Ti)

Ubat Puru

Serat

Kingkimut

Ingkakahas

Kaliabas (P), Liabas (Ka)

Solovondo (?)

Usod-usod

Sondotnulogo

Kutang (Kg)

Pongongondog

Pagawangan

Onininsil

Omnompik (Ta)
Collection No.

SAN 138287

SAN 138275

SAN 138285

$-$

FRCSE 420

FRCSE 389

SAN 142048

SAN 138284

SAN 238288

SAN 138282

SAN 138277

FRCSE 423

FRCSE 411

SAN 138230

$-$

$-$

FRCSE 406

$-$
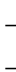
Botanical Name

Sida rhombifolia L. ${ }^{3}$

Sindora sp. ${ }^{2}$

Solanum torvum Sw. ${ }^{\star}$

Spatholobus cf. gyrocarpus Benth in Miq. *

Stachytarpheta jamaicensis (L.) Vahl.2

Stenochlaena palustris (Brum) Bedd²

Stephania corymbosa (BI) Wel.P. ${ }^{3}$

Strychnos ignatii Berg. ${ }^{1}$

Synedrella nodiflora (L) Gaertn. ${ }^{3}$

Tetrastigma diepenhostii (Mq)Latiff ${ }^{3}$

Tinospora crispa (L) Hook.f. \& Thomson*

Trichosanthes cucumerina L. ${ }^{3}$

Urena lobata L. ${ }^{3}$

Urophyllum nigricans Warnh. ${ }^{3}$

Uvaria grandiflora Roxb. ${ }^{3}$

Vitis trifolia (L.) Domin. ${ }^{1}$

Zea mays L. ${ }^{3}$

\section{Murut Name}

Dalupang (P)

Talikakasam

Lintahun (Ka)

Ramus

Indalupang

Kuraunolot

Tataga Do Sangi

Daramatin (Ti)

Molisun Mamulal (Ti)

Injilokot (Ti)

Nolilitan (Ti)

Susumoloi

Halai (Ka)
Collection No.

FRCSE 410

FRCSE 396

FRCSE 418

SAN 138292

SAN 138286

FRCSE 425

SAN 138270

\section{APPENDIX 2: PLANT FAMILIES MOST COMMONLY USED FOR MEDICINAL PURPOSES.}

Euphorbiaceae (10 spp.); Leguminosae (6 spp.); Rubiaceae (5 spp.); Araceae (4 spp.); Gramineae (4 spp.); Menispermaceae (4 spp.); Annonaceae (3 spp.); Lauraceae (3 spp.); Moraceae (3 spp.); Malvaceae (3 spp.); Agavaceae (2 spp.); Amaranthaceae (2 spp.); Labiatae (2 spp.); Palmae (2 spp.) and Piperaceae (2 spp.).

\section{One species each:}

Acanthaceae, Anarcadiaceae, Apocynaceae, Aristolochiaceae, Asteraceae, Begoniaceae, Bixaceae, Bromeliaceae, Cecropiaceae, Combretaceae, Costaceae, Cucurbitaceae, Dilleniaceae, Dipterocarpaceae, Flacourtiaceae, Flagellariaceae, Gesneriaceae, Guttiferae, Hypoxidaceae, Meliaceae, Myrtaceae, Oleaceae, Proteaceae, Rutaceae, Simaroubaceae, Solanaceae, Vitaceae, Zingiberaceae

\footnotetext{
$\mathrm{P}=$ Paluan, $\mathrm{Ka}=$ Kalabakan, $\mathrm{Ke}=$ Keningau, $\mathrm{Ti}=$ Timugon and $\mathrm{Ta}=$ Tagal

$1=$ Collected by Guntavid (1992) $\quad 2$ = Fasihuddin \& Hasmah (1992)

$3=$ Collected by Kulip (2001) * $\quad$ = Collected by all authors
} 